\title{
Pathogenesis of Non-alcoholic Steatohepatitis and Its Potential Therapeutic Strategies
}

\author{
Yoshihiro Ogawa, Takayoshi Suganami, Michiko Itoh, and Miyako Tanaka
}

\begin{abstract}
Non-alcoholic steatohepatitis (NASH) is closely associated with progression to liver cirrhosis and hepatocellular carcinoma. We reported that melanocortin 4 receptor-deficient mice (MC4R-KO mice), when fed a high-fat diet, provide a novel rodent model of NASH. Recently, we have identified a unique histological feature termed "hepatic crown-like structures" (hCLS) in the livers of MC4R-KO mice and NASH patients. In hCLS, CD11c-positive macrophages aggregate to surround hepatocytes with large lipid droplets, similar to the "crownlike structure (CLS)" described in obese adipose tissue. Interestingly, we have recently reported that macrophage-induced C-type lectin (Mincle) is involved in CLS formation and fibrogenic gene expression in obese adipose tissue, suggesting the pathophysiologic role of CLS in obesity-induced adipose tissue fibrosis. Collectively, our data provide evidence that hCLS serves as an origin of hepatic inflammation and fibrosis during the progression from simple steatosis to NASH and thus helps in elucidation of the pathogenesis of NASH, pursuit of specific biomarkers, and evaluation of potential therapeutic strategies.
\end{abstract}

Keywords Adipose tissue $\bullet$ Crown-like structure $\bullet$ Fibrosis $\bullet$ Inflammation $\bullet$ Liver - Macrophage-inducible C-type lectin (Mincle) - Metabolic syndrome • Nonalcoholic steatohepatitis (NASH) • Parenchymal cells $\bullet$ Stromal cells

\footnotetext{
Y. Ogawa $(\varangle)$

Department of Molecular Endocrinology and Metabolism, Graduate School of Medical and Dental Sciences, Tokyo Medical and Dental University, Tokyo 113-8510, Japan

Japan Agency for Medical Research and Development, CREST, Tokyo 100-0004, Japan e-mail: ogawa.mem@tmd.ac.jp

T. Suganami

Department of Organ Network and Metabolism, Graduate School of Medical and Dental Sciences, Tokyo Medical and Dental University, Tokyo 113-8510, Japan

Japan Science and Technology Agency, PRESTO, Tokyo, Japan

M. Itoh • M. Tanaka

Department of Organ Network and Metabolism, Graduate School of Medical and Dental Sciences, Tokyo Medical and Dental University, Tokyo 113-8510, Japan

K. Nakao et al. (eds.), Innovative Medicine, DOI 10.1007/978-4-431-55651-0_12
} 


\section{Introduction}

The metabolic syndrome is defined as a combination of visceral fat obesity, impaired glucose metabolism, atherogenic dyslipidemia, and blood pressure elevation, all of which are independent risk factors for atherosclerotic diseases. The molecular basis for the clustering of such independent risks of atherosclerosis is not currently unclear, with visceral fat obesity considered most important. There is considerable evidence that lifestyle diseases are a tissue-remodeling disease. Indeed, obese adipose tissue is characterized by adipocyte hypertrophy, increased angiogenesis and infiltration of immune cells such as macrophages and lymphocytes, tissue fibrosis, and increased production of proinflammatory adipokines [1]. The functional and morphological changes observed in obese adipose tissue are very similar to those described in vascular remodeling during the process of atherosclerosis. We have called the dynamic changes in obese adipose tissue "adipose tissue remodeling" and have been interested in the molecular basis underlying adipose tissue remodeling and its pathophysiological implication in metabolic diseases.

\section{Interaction Between Adipocytes and Macrophages in Obese Adipose Tissue}

We and others previously reported that during the progression from visceral fat obesity to a variety of lifestyle diseases, hypertrophied adipocytes can secrete a number of cytokines and chemokines such as monocyte chemoattractant protein-1 (MCP-1) to stimulate the recruitment of C-C chemokine receptor type 2 (CCR2)positive monocytes into obese adipose tissue as proinflammatory M1 macrophages. Once infiltrated, macrophages may be activated in response to saturated fatty acids released from hypertrophied adipocytes and produce a large amount of proinflammatory cytokines such as tumor necrosis factor- $\alpha$ (TNF- $\alpha$ ), which, in turn, augments the inflammatory response and adipocyte lipolysis to increase the release of fatty acids in hypertrophied adipocytes. These observations led us to speculate that there is an intimate crosstalk between parenchymal cells, adipocytes, stromal cells, and macrophages, which establishes a vicious cycle that augments obesity-induced adipose tissue inflammation [2, 3]. Our data also suggest that saturated fatty acids serve as a naturally occurring ligand for Toll-like receptor 4 (TLR4) complex, a well-known pathogen sensor expressed in infiltrating macrophages [3].

As the site of crosstalk between adipocytes and macrophages, there is a unique histological feature termed a "crown-like structure" (CLS) in obese adipose tissue [4], which is composed of dead parenchymal adipocytes surrounded by macrophages.

In CLS, macrophages are considered to scavenge the residual lipid droplets of dead or dying adipocytes. It is likely that the adipocyte-macrophage interaction can stimulate adipocyte lipolysis and thus increase the release of free fatty acids, particularly saturated fatty acids, from obese adipose tissue, which may be accumulated 
in non-adipose tissue as ectopic fat, thereby inducing a variety of metabolic effects called lipotoxicity [5].

\section{Development of a Novel Rodent Model of NASH}

Non-alcoholic fatty liver disease (NAFLD) is defined as increased accumulation of lipids in the liver without a history of excess alcohol consumption. It is considered the hepatic manifestation of the metabolic syndrome. Non-alcoholic steatohepatitis (NASH), the most aggressive subset of NAFLD, is closely associated with the development of liver cirrhosis and hepatocellular carcinoma. The pathogenesis of NASH, the well-known "two-hit hypothesis", has been proposed for many years; however, the detailed mechanism of disease progression from simple steatosis to NASH is currently unclear, partly because there are no appropriate animal models that reflect the liver condition of human NASH and partly because liver biopsy is required for its definite diagnosis.

Melanocortin-4 receptor (MC4R) is a seven-transmembrane $\mathrm{G}$ protein-coupled receptor that is expressed in the hypothalamic nuclei implicated in the regulation of food intake and body weight. Its pathogenic mutations are the most common known monogenic cause of human obesity. We recently reported that MC4R-deficient (MC4R-KO) mice develop a liver condition similar to human NASH when fed a high-fat diet (HFD) or a Western diet (WD), which is associated with obesity, insulin resistance, and dyslipidemia [6]. Notably, they exhibited enhanced adipose tissue inflammation, which may contribute to excessive lipid accumulation and enhanced fibrosis in the liver. Moreover, they developed well-differentiated HCC for a prolonged time. Our data suggest that MC4R-KO mice would provide a novel rodent model of NASH with which to investigate the sequence of events that encompass diet-induced obesity, hepatic steatosis, liver fibrosis, and HCC. Histologically, they showed hepatocyte ballooning degeneration, increased infiltration of inflammatory cells such as macrophages and lymphocytes, and pericellular fibrosis, all of which are hallmarks of human NASH. Immunohistochemical staining with $\alpha$-smooth muscle action $(\alpha \mathrm{SMA})$ revealed that hepatic stellate cells are activated in the liver from MC4R-KO mice even before the onset of fibrosis.

\section{Identification of Hepatic Crown-Like Structure (hCLS)}

During the course of obesity, MC4R-KO mice developed a number of CLS in the adipose tissue. We found bizarre microstructures in livers from MC4R-KO mice, which are very similar to CLS in obese adipose tissue [7]. We have called them "hepatic crown-like structures" (hCLS), where macrophages aggregate to surround hepatocytes with large lipid droplets. Although there was no significant difference in the number of F4/80-positive macrophages between the genotypes, the number of 
hCLS was increased in MC4R-KO mice relative to wild-type mice, suggesting that the distribution of macrophages was altered in the livers from MC4R-KO mice. Importantly, there was a significant correlation between the number of hCLS and the extent of liver fibrosis. These observations, taken together, suggest that hCLS rather than macrophages themselves are associated with the development of liver fibrosis during the progression from simple steatosis to NASH.

To further explore the functional significance of hCLS in the development of liver fibrosis, we depleted macrophages in livers from MC4R-KO mice by the clodronate liposome method. In MC4R-KO mice, macrophages scattered in the liver were effectively depleted, although those in hCLS appeared to be intact. Accordingly, treatment with clodronate liposome significantly reduced the F4/80-positive areas in the liver from MC4R-KO mice. However, there was no significant change in the numbers of hCLS and $\alpha$ SMA-positive cells after the treatment. In this setting, treatment with clodronate liposome resulted in a significant reduction of expression of inflammatory and fibrogenic genes in the liver from wild-type mice. By contrast, in MC4R-KO mice, there was no significant change in the gene expression after the treatment. These observations suggest that hCLS macrophages are resistant to the treatment and that hCLS is functionally associated with hepatic inflammation and fibrosis in NASH liver. Immunofluorescence and electron microscopy analyses revealed that hCLS is composed of CD11c-positive macrophages and dead parenchymal hepatocytes with large lipid droplets, and is spatially associated with $\alpha$ SMA-positive activated stellate cells and collagen deposition, which support the notion that hCLS is involved in the development of hepatic inflammation and fibrosis.

We also found that hCLS occurs in patients with NASH or even in those with simple steatosis in proportion to the extent of liver fibrosis. Importantly, there was no such increase in hCLS in patients with chronic viral hepatitis, suggesting that hCLS is rather specific to metabolic liver diseases.

Figure 1 illustrates the role of hCLS in the pathogenesis of NASH. Increased flux of free fatty acids from visceral adipose tissue to the liver via the portal vein as a result of adipose tissue inflammation can cause simple steatosis. Parenchymal hepatocytes that are being overloaded with lipid and are thus dead or dying are surrounded by macrophages to form hCLS in the fatty liver. In the NASH liver, macrophages can interact with and engulf dead hepatocytes within hCLS, thereby activating fibrogenic cells to stimulate hepatic fibrosis as an adaptive repair response to tissue injury. It is, therefore, likely that hCLS serves as an origin of hepatic inflammation and fibrosis during the progression from simple steatosis to NASH [7].

\section{Macrophage-Induced C-Type Lectin (Mincle)}

Macrophage-induced C-type lectin (Mincle) is a type II transmembrane $\mathrm{Ca}^{+}$dependent carbohydrate binding lectin. It is expressed abundantly in macrophages, where it is induced in response to LPS. Recent studies show that Mincle is a pathogen sensor that recognizes the mycobacterial glycolipid trehalose dimycolate 




Fig. 1 Possible role of hCLS during the progression from normal healthy liver and simple steatosis to NASH

(TDM) [8]. More interestingly, it can also recognize an endogenous ligand that is released from dead cells as a danger signal [9]. We previously reported that during the interaction between adipocytes and macrophages, saturated fatty acids can induce Mincle expression in macrophages through the activation of the TLR4/ nuclear factor $\kappa \mathrm{B}(\mathrm{NF}-\kappa \mathrm{B})$ pathway [10].

Mincle mRNA expression is induced in obese adipose tissue, which occurs mostly in the stromal vascular fraction. FACS analysis revealed that Mincle is induced in the infiltrating macrophages in obese adipose tissue; a substantial fraction of the macrophages is Mincle-positive. By in situ hybridization analysis, we found that Mincle mRNA expression is restricted to CLS macrophages in obese adipose tissue. We also found that Mincle expression is induced in the adipose tissue of obese humans [10]. There is a significantly positive correlation between Mincle mRNA levels and BMI, suggesting the role of Mincle in the pathogenesis of obesity. These observations suggest that Mincle plays a role in adipose tissue remodeling.

\section{Role of Mincle in Adipose Tissue Remodeling and Ectopic Fat Accumulation}

To explore the role of Mincle in adipose tissue remodeling in vivo, we examined the metabolic phenotype of Mincle-KO mice during the HFD feeding. There was no apparent difference in body weight between the genotypes throughout the 
experimental period. At 8 weeks of HFD feeding, when macrophages were not massively infiltrated, there was no significant difference in adipose tissue and liver weight between the genotypes. Notably, at 16 weeks of HFD feeding, when macrophages were massively infiltrated, Mincle-KO mice showed increased adipose tissue weight with a reciprocal reduction of liver weight. Histological analysis of the adipose tissue revealed that adipocytes seemed to be enlarged in Mincle-KO mice in response to the HFD feeding. Although there was no significant difference in F4/80positive macrophages between the genotypes, the number of CLS was significantly reduced in Mincle-KO mice. Since the number of hCLS is more positively correlated with the extent of liver fibrosis than that of macrophages in MC4R-KO mice [7], we examined adipose tissue fibrosis by Masson trichrome staining. There was extensive interstitial fibrosis in the epididymal fat depot from wild-type mice, which was markedly attenuated in Mincle-KO mice together with a significant reduction of collagen deposition. Interestingly, there was no significant difference in collagen deposition in the subcutaneous fat depot between the genotypes; this may be partly because Mincle expression is not markedly increased in obese subcutaneous fat depots, where macrophage infiltration was not so remarkable. Thus, Mincle-KO mice exhibited increased adipose tissue weight with increased adipocyte cell size with significant reduction of CLS and reduced adipose tissue fibrosis.

We next examined the liver phenotypes of Mincle-KO mice and found that hepatic steatosis is markedly attenuated in Mincle-KO mice. Accordingly, liver TG content and serum ALT concentrations were significantly reduced in Mincle-KO mice. We also examined glucose metabolism and insulin sensitivity by glucose and insulin tolerance tests and confirmed improved glucose metabolism and insulin sensitivity in Mincle-KO mice.

During the paracrine interaction between adipocytes and macrophages, saturated fatty acids, which are released via the macrophage-induced adipocyte lipolysis, are able to induce Mincle in the infiltrating CLS macrophages through the TLR4/NF- $\kappa B$ pathway $[10,11]$. Mincle may recognize as-yet-unidentified endogenous ligands released from dead adipocytes as a danger signal, thereby stimulating adipose tissue fibrosis. In Mincle-KO mice, where adipose tissue fibrosis is attenuated, adipocytes can be hypertrophied enough to store lipid, which may reduce ectopic fat accumulation in the liver. Accordingly, our data suggest that Mincle plays a critical role in the formation of CLS and adipose tissue fibrosis during the course of obesity, which may reduce lipid-storage capacity in adipose tissue and enhance ectopic lipid accumulation [11].

\section{Summary}

Our data suggest that CLS and hCLS provide the unique microenvironment where dead or dying parenchymal and stromal cells crosstalk in close proximity in vivo (Fig. 2). In NASH liver, parenchymal hepatocytes, when they are overloaded with lipids and thus dead or dying, report their dysfunctional state to the adjacent stromal 


\section{hCLS}
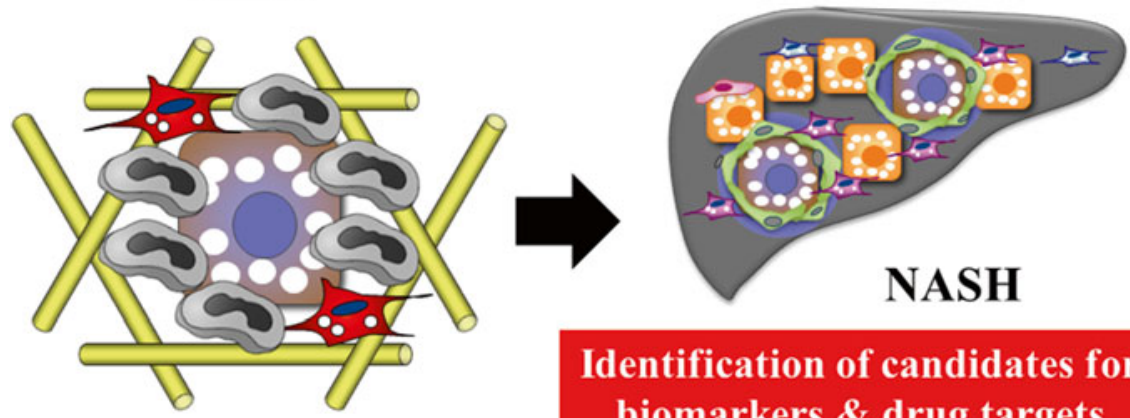

Identification of candidates for biomarkers \& drug targets

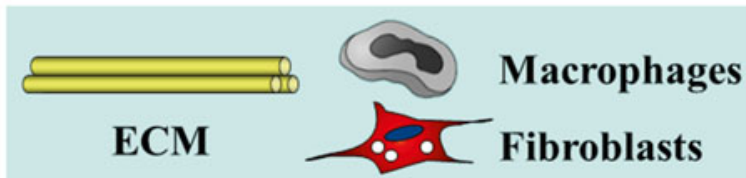

Fig. 2 Detailed analysis of hCLS helps identify candidate molecules to be used as biomarkers and/or drug targets specific for NASH

cells such as macrophages and fibroblasts via multiple danger signals or dying messages. Given that Mincle, when induced in macrophages in CLS, plays a critical role in the development of adipose fibrosis, it is tempting to speculate that Mincle is also involved in the progression of hepatic fibrosis. Detailed analysis of hCLS may provide a clue to discover candidate molecules to be used as biomarkers and/or drug targets specific for NASH.

Acknowledgments We thank the members of the Ogawa laboratory for discussions. Part of this study has been supported by the Uehara Memorial Foundation.

Open Access This chapter is distributed under the terms of the Creative Commons Attribution Noncommercial License, which permits any noncommercial use, distribution, and reproduction in any medium, provided the original author(s) and source are credited.

\section{References}

1. Suganami T, Ogawa Y (2010) Adipose tissue macrophages: their role in adipose tissue remodeling. J Leukoc Biol 88:33-39

2. Suganami T, Nishida J, Ogawa Y (2005) A paracrine loop between adipocytes and macrophages aggravates inflammatory changes: role of free fatty acids and tumor necrosis factor $\alpha$. Arterioscler Thromb Vasc Biol 25:2062-2068

3. Suganami T, Tanimoto-Koyama K, Nishida J, Itoh M, Yuan X, Mizuarai S, Kotani H, Yamaoka S, Miyake K, Aoe S, Kamei Y, Ogawa Y (2007) Role of the Toll-like receptor 4/NF-кB pathway 
in saturated fatty acid-induced inflammatory changes in the interaction between adipocytes and macrophages. Arterioscler Thromb Vasc Biol 27:84-91

4. Cinti S, Mitchell G, Barbatelli G, Murano I, Ceresi E, Faloia E, Wang S, Fortier M, Greenberg AS, Obin MS (2005) Adipocyte death defines macrophage localization and function in adipose tissue of obese mice and humans. J Lipid Res 46:2347-2355

5. Suganami T, Tanaka M, Ogawa Y (2012) Adipose tissue inflammation and ectopic lipid accumulation. Endocr J 59:849-857

6. Itoh M, Suganami T, Nakagawa N, Tanaka M, Yamamoto Y, Kamei Y, Terai S, Sakaida I, Ogawa Y (2011) Melanocortin-4 receptor-deficient mice as a novel mouse model of nonalcoholic steatohepatitis. Am J Pathol 179:2454-2463

7. Itoh M, Kato H, Suganami T, Konuma K, Marumoto Y, Terai S, Sakugawa H, Kanai S, Hamaguchi M, Fukaishi T, Akiyoshi K, Komohara Y, Takeya M, Sakaida I, Ogawa Y (2013) Hepatic crown-like structure: a unique histological feature in non-alcoholic steatohepatitis in mice and humans. PLoS One 8:e82163

8. Ishikawa E, Ishikawa T, Morita YS, Toyonaga K, Yamada H, Takeuchi O, Kinoshita T, Akira S, Yoshikai Y, Yamasaki S (2009) Direct recognition of the mycobacterial glycolipid, trehalose dimycolate, by C-type lectin Mincle. J Exp Med 206:2879-2888

9. Yamasaki S, Ishikawa E, Sakuma M, Hara H, Ogata K, Saito T (2008) Mincle is an ITAMcoupled activating receptor that senses damaged cells. Nat Immunol 9:1179-1188

10. Ichioka M, Suganami T, Tsuda N, Shirakawa I, Hirata Y, Satoh-Asahara N, Shimoda Y, Tanaka M, Kim-Saijo M, Miyamoto Y, Kamei Y, Sata M, Ogawa Y (2011) Increased expression of macrophage-inducible C-type lectin in adipose tissue of obese mice and humans. Diabetes 60:819-826

11. Tanaka M, Ikeda K, Suganami T, Komiya C, Ochi K, Shirakawa I, Hamaguchi M, Nishimura S, Manabe I, Matsuda T, Kimura K, Inoue H, Inagaki Y, Aoe S, Yamasaki S, Ogawa Y (2014) Macrophage-inducible C-type lectin underlies obesity-induced adipose tissue fibrosis. Nat Commun 5:e4982 